\title{
Maternal Mortality: Case Reviews and Lessons Learned from the Global Partnerships and the Maternal Fetal Medicine Scientific Forum on Global Health
}

\author{
Dotun Ogunyemi*, Gabriel Ganyaglo, Godfrey Mugyenyi \\ Associate Dean of faculty Affairs, California University of Science \& Medicine, USA
}

Submission: August 14, 2019; Published: September 05, 2019

*Corresponding author: Dotun Ogunyemi, Associate Dean of faculty Affairs, Multicultural Diversity \& Inclusion, Professor of Medical Education; Obstetrics \& Gynecology, California University of Science \& Medicine, 217 E Club Center Dr suite a, San Bernardino, CA 92408.

\begin{abstract}
We describe collaboration between obstetrician leaders in low resource countries and MFM specialists in promoting a systematic approach to maternal mortality cases reviews and targeting feasible quality improvement projects. This effort cumulated in the presentation and reviews of representative cases of maternal mortality by emerging obstetrician leaders from Ghana, Uganda, India and Haiti at the Global Health Scientific Forum of Society of Maternal Fetal Medicine Annual Meeting in 2012. In addition to large-scale initiatives, groups of maternal healthcare providers can develop similar global partnership that can facilitate the goal of reducing global maternal mortality.
\end{abstract}

Abbreviations: MDG: Millennium Development Goals; ACOG: American college of Obstetricians and Gynecology; US: United States; MFM: Maternal-Fetal-Medicine; SMFM: Society of Maternal Fetal Medicine; RCA: Root Cause Analysis

\section{Introduction}

Maternal mortality is defined as the death of a woman while pregnant or within 365 days of the end of pregnancy, from any cause related to or aggravated by the pregnancy or its management, but not from accidental or incidental causes [1,2]. The Millennium Development Goals (MDG) 5 signed by 189 heads of state, calls for a $75 \%$ reduction in the global maternal mortality 1990 and 2015 [3]. In 2011, the world maternal mortality was reported to have decreased from 409,100 in 1990 to 256,300 deaths, a 38\% decrease, with only 13 countries noted to be on track to achieve MDG5 [3]. Overall progress in decreasing maternal mortality has been inadequate and inconsistent, especially in low resourced countries. This has resulted in urgent efforts to tackle maternal morality such as the United Nations Global Strategy for Women's and Children's Health; Saving Mothers, which is a partnership between American college of Obstetricians and Gynecology (ACOG), United States (US) and Norwegian governments focusing on the critical 24 hours of labor \& delivery [4]. However, more interventions are needed by more maternal health organizations, institutions and physicians' stakeholders to decrease maternal mortality and achieve MDG5.

Purpose

The purpose of this report is to describe a collaborative process between obstetrician leaders in low resource countries and US Maternal-Fetal-Medicine (MFM) specialists in promoting a systematic approach to maternal mortality cases reviews, identifying system issues that impact safe care, targeting feasible quality improvement projects that may lead to a reduction of maternal mortality in developing countries, and the presentation of representative cases of maternal mortality for discussion and review at the Global Health Scientific Forum of Society of Maternal Fetal Medicine Annual Meeting in 2012.

Our goal was also to suggest feasible educational programs based on our experiences that can be adopted by physicians in resource-high countries who are interested in supporting and collaborating with counterparts in low-resource countries in the effort to develop a culture of safety, in-depth blame free maternal mortality reviews and opportunities to improve the health care delivery system in order to decrease maternal deaths.

\section{Process}

This program occurred as a result of the interest and efforts of the members of the Society of Maternal Fetal Medicine (SMFM) sub-committee on Global Health. This committee is committed to improving the health of women and children in underserved international communities. The purpose was to identify effective strategies to address maternal mortality in underserved communities using the following processes. 


\section{Global Journal of Reproductive Medicine}

1) The first step required that MFM specialists interested in global health identify and develop a professional, collaborative and mentoring relationship with an emerging physician leader from a facility in a low resource country.

2) The next step ensured that the MFM specialists sustain and maintain an ongoing relationship with the low-resource emerging leader through bilateral visits, consultations and electronic teleconferences, emails and other communication methods.

3) Best practice for root cause analysis (RCA) for maternal mortality review was promoted. Root cause analysis (RCA) is a structured method used to analyze serious adverse by identifying underlying problems that increase the likelihood of errors while avoiding the trap of focusing on mistakes by individuals. The goal of RCA is to identify both active errors (errors occurring at the point of interface between humans and a complex system) and latent errors (the hidden problems within health care systems that contribute to adverse events). RCA generally involves a multidisciplinary team follow a protocol that begins with data collection and reconstruction of the event in question through record review and participant interviews. The ultimate goal of RCA is to prevent future harm by eliminating the latent errors that underlie adverse events $[5,6]$.

4) The low-resource leaders were invited to an inaugural scientific forum on Global Maternal Mortality at the annual meeting of the Society for Maternal Fetal Medicine in February 2012. Each member presented a case history that was representative of one of the key drivers of maternal mortality, followed by root cause analyses with US MFM experts facilitating discussions in a global context. The cases were presented using the 3-delay model (delay in making decisions to seek medical care, delay in reaching an appropriate facility, delay in receiving appropriate care on reaching facility) [7], which recognizes that timely and adequate treatment for obstetric complications are major factors in reducing maternal deaths.

5) A fishbone analysis was also developed for each case presented. A fishbone diagram, also called a cause and effect diagram or Ishikawa diagram, is a visualization tool for categorizing the potential causes of a problem in order to identify its root causes [8,9].

6) Post-conference, MFM experts-maintained contact and support with the low resource countries with continued support for the confidential review system and a focus on implementing feasible quality improvement projects that could improve safe care and decrease maternal mortality.

\section{Cases}

The four emerging physician leaders were from Uganda, Ghana Haiti, and India. These were the countries that the MFM specialists were able to develop sustainable professional relationships with local physician leaders. These relationships had been ongoing for approximately 2 years prior to the SMFM conference. The cases presented at the Global Health Scientific Forum of the Society of Maternal Fetal Medicine reflected the leading direct causes of maternal death - septic abortion, obstetrical hemorrhage, obstructed labor and preeclampsia.

\section{Septic abortion, accra, ghana}

A thirty-seven-year-old Para 5 with three months amenorrhea procured herbal preparations from a street vendor to terminate her pregnancy in secrecy. This was unsuccessful, so she obtained an illicit pregnancy termination at an unidentified private clinic. On post-operative day 4 , she developed severe abdominal pain and her husband took her to a district hospital. She did not reveal any information about a pregnancy nor an unsafe abortion attempt. She was evaluated, but a pelvic examination was not done. She was admitted for presumed pneumonia with sepsis and given antibiotics, fluids, and 1 unit of blood. On hospital day 3 , she developed fever, productive cough with vaginal bleeding and passage of tissue. A diagnosis of pelvic abscess was made; patient had referred her to the tertiary hospital approximately 30 kilometers away by public transport.

Upon arrival, she was febrile, jaundiced, hypotensive and anuric. She had bronchial breath sounds, tender and distended abdomen, 12 weeks sized tender uterus, and dilated cervix with offensive products of conception. The diagnosis was septicemia secondary to septic incomplete abortion and pneumonia. Confronted with the physical findings, patient revealed the history. She did not volunteer information about her termination previously because of the presence of her husband. She was resuscitated with normal saline, oxygen and broad-spectrum antibiotics. Eight hours later, she was taken to the operating room for uterine evacuation. The cervix was edematous, friable with a laceration but no evidence of uterine perforation. Very offensive and copious placental tissue was removed.

Post-evacuation, she was critically ill with unstable blood pressure, tachycardia and oliguria. Intravenous antibiotics and blood transfusion were continued. Ten hours post-evacuation she had a cardiac arrest but recovered after cardiopulmonary resuscitation. She deteriorated and was declared clinically dead about ninety-six minutes after the cardiac arrest. Postmortem diagnosis was bilateral lobar pneumonia with septic abortion.

Septic abortion fishbone and root cause analysis: A fish bone diagram Figure 1 highlights the problems identified during the discussion that were considered potential root causes of her death. These included unwanted pregnancy, inadequate access to and knowledge about family planning services. By not disclosing her attempts at pregnancy termination she contributed to a delay in diagnosis, although an incomplete assessment was performed at the district hospital. Once transferred to the referral hospital, resources were limited. Lack of formal education and male 
dominance are also major factors in this case of mortality from the complications of an unsafe abortion. At least one in five Ghanaian women age 15-49 years have no formal education, compared to one in eight for Ghanaian men.

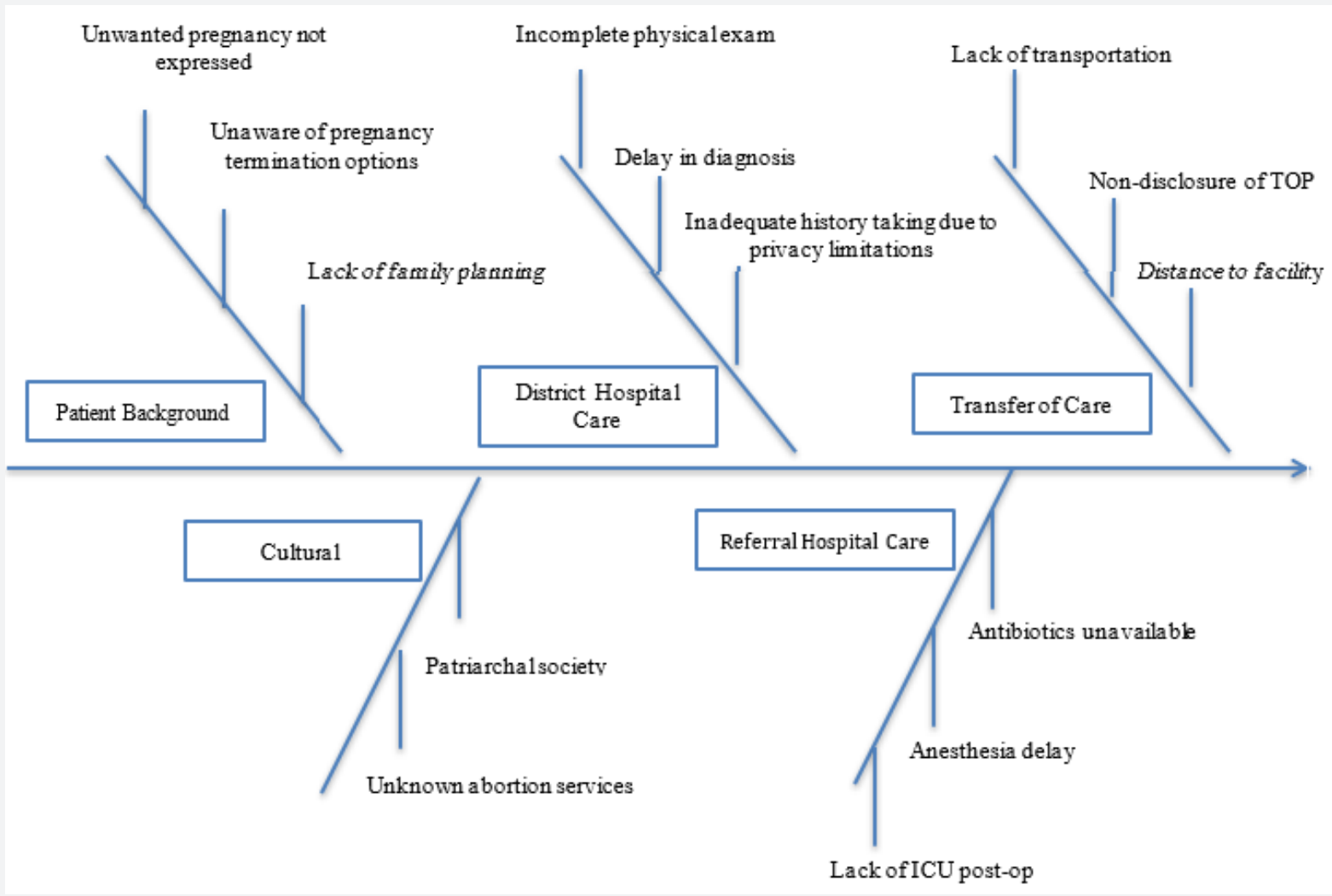

Figure 1: Images of sentinel lymph nodes obtained by nuclear medicine.

The cultural issue of male dominance in the Ghanaian society further widens the gap in equitable access to care $[10,11]$. Preventive processes that have been identified and implemented include developing a feedback system, improving access to and awareness of family planning services, and improve awareness of Ghana's abortion laws to health providers. Long-term projects that involve government policy and major funding include increasing the education of girls, and the empowerment of women politically and economically. Also required are health care resources such as ICU, availability of antibiotics and skilled health care providers.

\section{Obstructed labor, Mbarara, Uganda}

A 28-year-old primigravid HIV positive Rwandese refuge was admitted following referral by a primary health facility for obstructed labor with fetal death at 39 weeks of gestation. She was not on antiretroviral medications and had an unknown CD4 count and viral load. She had labored for three days at home. She started pushing at home under the care of a traditional birth attendant who sent her to the primary health facility because the baby had "failed to come." Subsequently, the primary health facility referred her to the regional hospital, which was 40 kilometers away, at which exploratory laparotomy revealed a lower segment uterine rupture, massive hemo-pyo-peritonium with the placenta in the abdominal cavity and extensive necrosis of the lower uterine segment and pelvic fascia. A macerated male stillbirth of 3.9 kilograms was delivered and total hysterectomy with bilateral internal iliac artery ligation done within 85 minutes of arrival. Four units of whole blood were transfused during the operation. The patient remained hypovolemic with tachycardia and poor tissue perfusion post operatively. She developed signs of pulmonary edema, had convulsions; deteriorated and expired on the fourth day of management. Cause of death was cardiopulmonary failure and septicemia resulting from ruptured uterus following neglected obstructed labor in the community. No autopsy was done.

Obstructed labor Fishbone and Root Cause Analysis: Figure 2 shows the fish bone diagram that highlights the root causes of this mortality. There was delay in making the decision to seek medical care, since the patient labored at home for more than 3 days. There was a crucial delay in reaching the appropriate facility because the initial health center was not equipped for critical obstetrical procedures and she had to be transported 40 kilometers to the tertiary center. There was also a delay in receiving appropriate management on arrival at tertiary center. A program implemented in this institution is a maternal death confidential inquiry audit which involves tracing all the way back to the deceased's home and holding discussions with all concerned. 


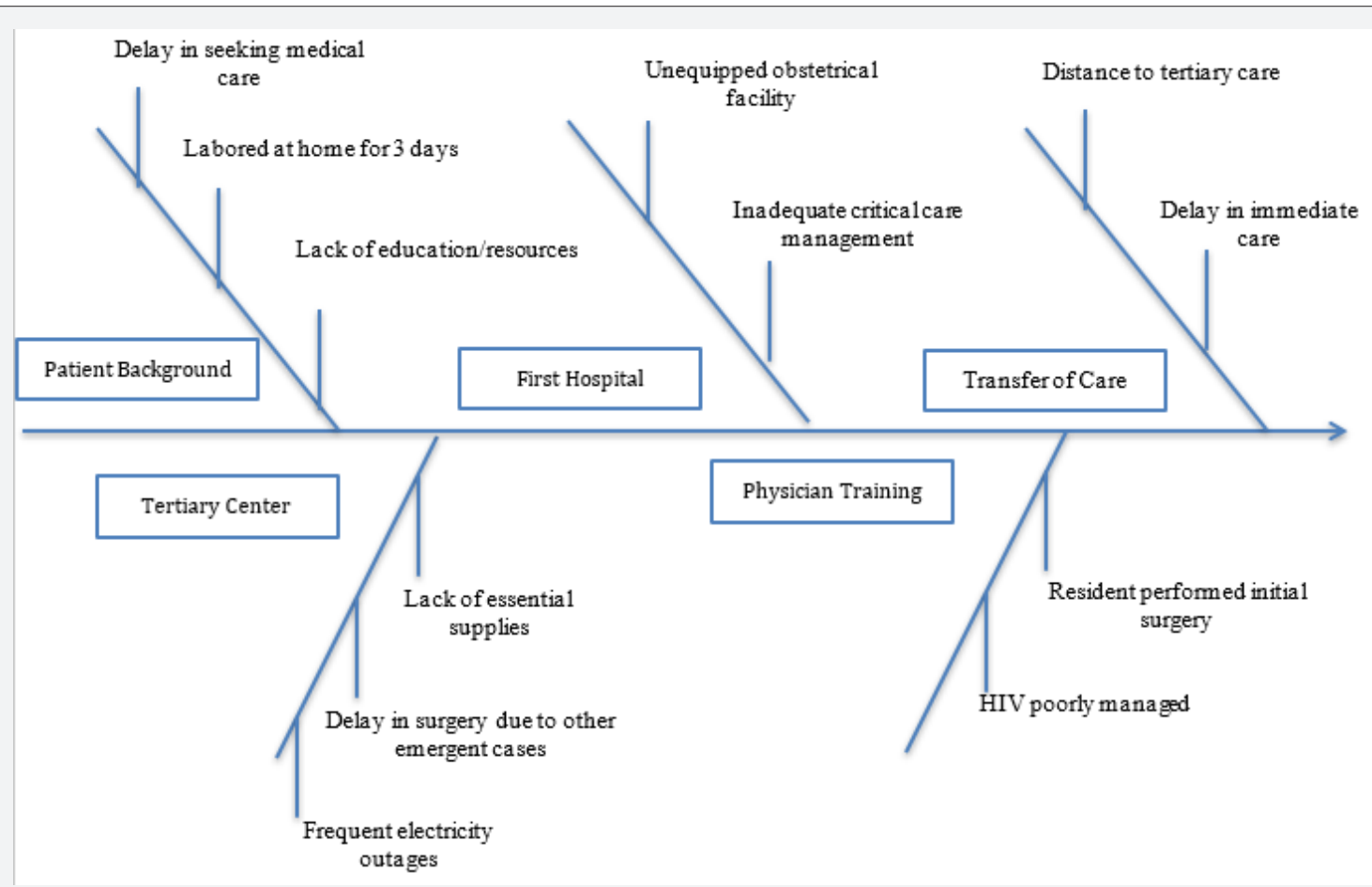

Figure 2: Fishbone Diagram for Case of Obstructed Labor and Maternal Mortality (UGANDA).

Discussions are arranged with the health workers directly involved in the management of the deceased mother, immediate family members, and community leaders. The final audit and review is utilized to recommend, advocate and implement programs to reduce maternal mortality. This institution in collaboration with counterparts from high resource institutions has initiated achievable quality improvement projects as requirements for the OBYN residents as a method to target systems deficits in care.

\section{Peripartum hemorrhage, madurai, india}

A multiparous 33-year-old, at 40 weeks of gestation with uneventful antenatal course was admitted at a primary facility in spontaneous labor and progressed well. She had a second stage of approximately ninety minutes complicated by maternal exhaustion and fetal bradycardia. Outlet forceps were applied and a healthy female baby weighing $3.4 \mathrm{~kg}$ was delivered. The episiotomy wound was sutured, and the cervix and vagina explored and found to be intact. There was no postpartum hemorrhage, and she was transferred to the post-partum unit. Approximately two-and-a-half hours later, patient was found unconscious by her attendant and could not be aroused. Patient was resuscitated and subsequently was referred to a tertiary care center for further management.

She was received 1-hour later in the Casualty unit of a regional hospital because transporting taxi-driver felt that the patient was deteriorating and would not make it to the designated tertiary center. Her pulse was feeble, blood pressure un-recordable, and patient was gasping. She was resuscitated with parenteral fluids and intubated. On abdominal examination, uterus was 24 weeks size and pushed towards the right side by a boggy mass extending from the bladder on the left side, halfway to the level of the umbilicus. Vaginal exam revealed normal cervix and minimal vaginal bleeding with fullness felt in the left fornix.

Ultrasound showed a suspected left side pelvic hematoma close to the uterus with a possible ruptured uterus. Laboratory results revealed bleeding coagulopathy with anemia, and she was appropriately transfused with fresh frozen plasma and packed red blood cells. Patient was taken for exploratory laparotomy approximately one hour after arrival. Intraoperative findings revealed a left broad ligament and a rupture of the left lateral uterine wall. Total abdominal hysterectomy left salpingooophorectomy and bilateral internal iliac artery ligation was performed. Postoperatively, patient was transferred to ICU. Packed cells, fresh frozen plasma and platelet transfusions were continued. While the altered coagulation profile was corrected, the hypotension persisted. Neurological evaluation revealed global cerebral damage. Patient developed repeated episodes of cardiac arrest and finally could not be revived. She expired approximately 24 hours after delivery.

Peripartum hemorrhage fishbone and root cause analysis: A fish bone diagram Figure 3 highlights the problems identified during the discussion that are considered potential root causes of the maternal mortality. In this postpartum hemorrhage case, there was no obvious external blood loss to alert the healthcare providers instead a significant volume of blood loss occurred intra-abdominally, leading to hemorrhagic shock and subsequent 


\section{Global Journal of Reproductive Medicine}

disseminated intravascular coagulation. There was inadequate monitoring of the fourth stage of labor. It is likely that if the pelvic hematoma was recognized earlier and appropriate surgical treatment was done immediately, the patient's life may have been saved. During transport to a higher referral center, there was a delay in reaching an appropriate facility, since a taxicab was transporting the patient to a tertiary center, when she became "moribund" and she was taken to a nearest hospital.

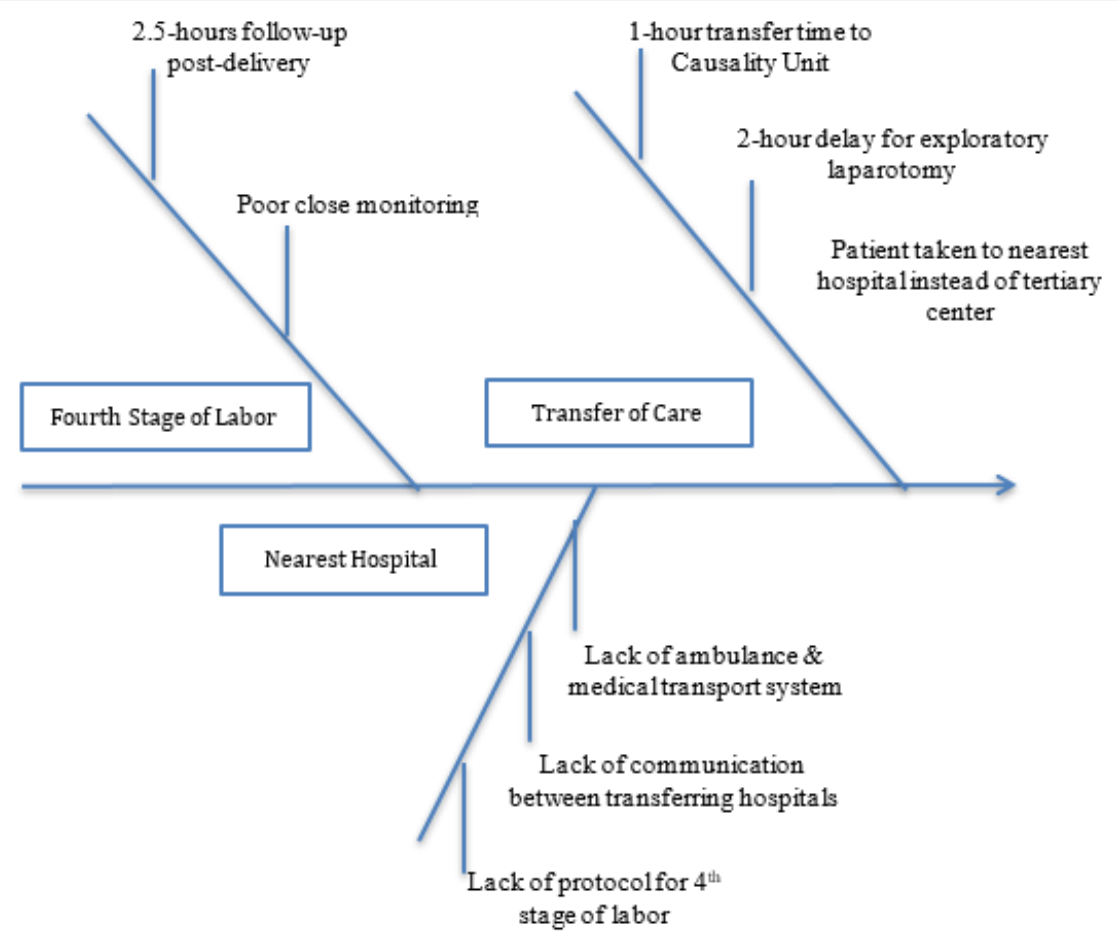

Figure 3: Fishbone Diagram for Case of Postpartum Pregnancy and Maternal MORTALITY (MADURAI, INDIA).

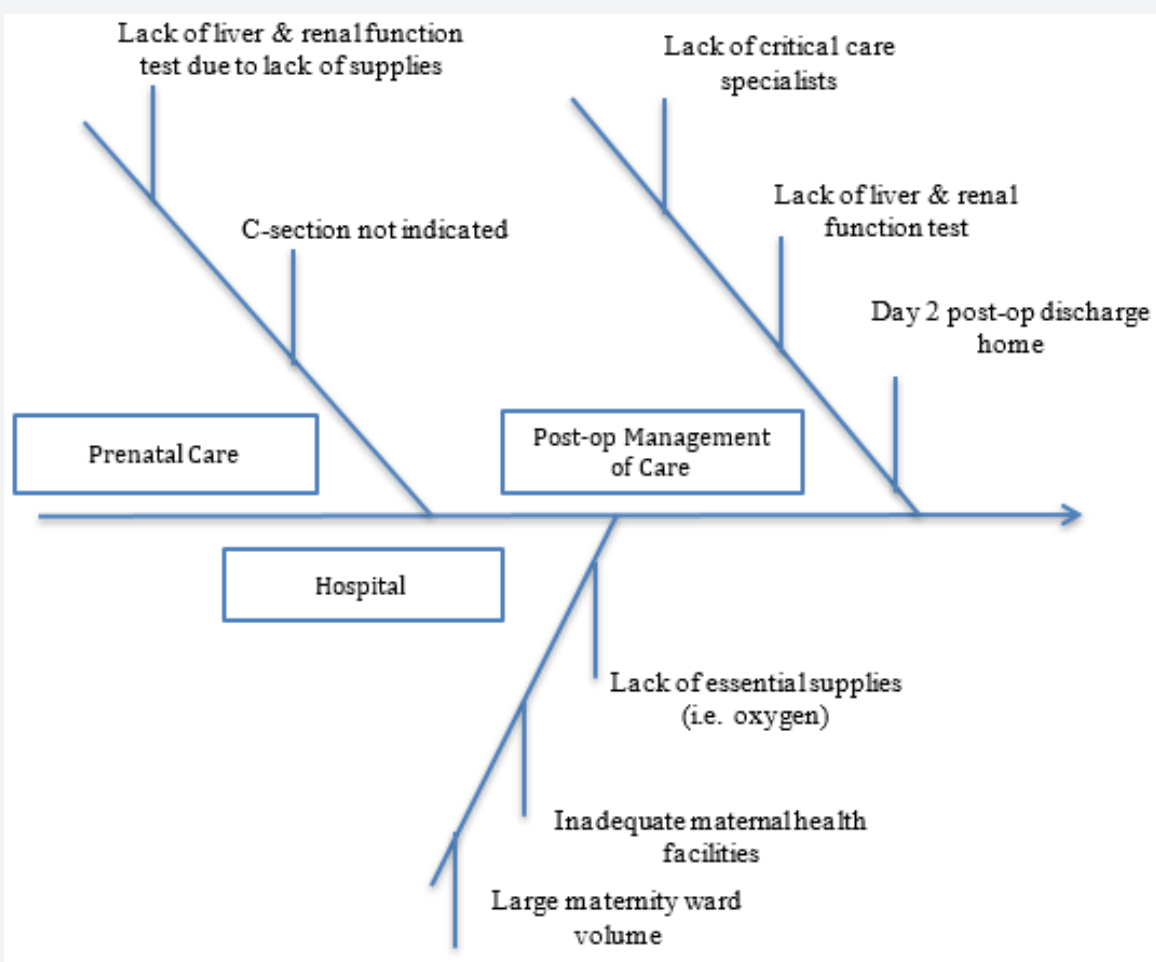

Figure 4: Fishbone Diagram for Case of Preeclampsia and Maternal Mortality (HAITI). 


\section{Global Journal of Reproductive Medicine}

\section{Preeclampsia, haiti}

A 37-year-old, primigravida at 39 weeks of gestation was referred because of new onset hypertension treated with methyldopa. Blood pressure was 170/120. Ultrasound showed a fetus, in cephalic presentation with estimated fetal weight less than the 10th percentile, normal amniotic fluid, umbilical artery Doppler with no end diastolic flow and the placenta had multiple lacuna. Platelets, renal function tests and liver function tests were not done because of a lack of reagents. A diagnosis of preeclampsia, intrauterine growth restriction and suspected placenta abruption was made. Blood pressure was controlled and stabilized with intravenous Apresoline. An uncomplicated cesarean section was performed because of the suspected abruption. She delivered a healthy female baby, birth weight 2580 grams with a normal placenta noted. Postpartum; her blood pressures stabilized to 140-130/80. She was asymptomatic with no complaints; renal and liver function tests were not done. On postpartum day 2 , her blood pressure was $140 / 80$, with normal clinical evaluation. She was discharged home on Methyldopa.

She presented to the Emergency Room the next day (postpartum day 3) with respiratory distress, epigastric pain, cough productive of whitish sputum, blood pressure of 220/120 and crackles on both lungs. The differential diagnoses entertained were pulmonary edema, pulmonary embolism and hepatic rupture. She was treated with Magnesium sulfate and Apresoline but died during resuscitation. An autopsy was not performed.

Preeclampsia fishbone and root cause analysis: A fish bone diagram Figure 4 highlights the problems identified during the discussion that are considered potential root causes of this mortality. This case highlights the contribution of inadequate management on arrival at a health facility to maternal mortality. The only indication for cesarean delivery was the ultrasonic abruption diagnosis. In this institution, patients with cesarean section are discharged on the 3rd post-operative day but because of high patient load and inadequate maternal care facilities, this patient was discharge home on the 2 nd post-operative day. Liver and renal function tests that might have demonstrated severe preeclampsia were not performed prior to discharge. RCA highlighted lack of resources such as critical care specialists; oxygen in the emergency room and laboratory test reagents.

\section{Discussion}

We have used four illustrative examples of maternal mortality to make specific points about obstetrical care in different international settings noting that these cases do not portend to describe all the common problems at each site or country. The death from unsafe abortion emphasizes the fact that despite Ghana's liberal abortion law, illicit abortion continues because of lack of public education, and inadequate resources [11]. The death from obstructed labor from Uganda highlights the pivotal role of traditional birth attendants and inadequate transportation infrastructure [12]. The case of unapparent postpartum hemorrhage from India; granted that this case may be challenging in any high resource country nevertheless emphasizes the importance of the fourth stage of labor and the need for close monitoring of the immediate postpartum period. The maternal mortality from severe preeclampsia from Haiti emphasizes inadequate maternity health care facilities and lack of ancillary resources.

Many of the drivers of maternal mortality such as transportation, facilities, drugs, equipment, skilled health care workers and cultural taboos are daunting and overwhelming in scope and enormity. Successful interventions would require major political will, policy changes, health-care infrastructure, largescale funding and fundamental cultural changes [13]. Therefore even though reviewing these maternal mortality cases may seem to be "hand-wringing", however our goal was to report feasible projects that can be undertaken by individual or small groups of obstetricians, MFM specialists or professional organization committees in collaboration with physicians in low-resource communities with the aim to reduce global maternal mortality.

The cases presented were based on professional mentoring relationships developed over time with specific physician leaders in the four countries. The MFM specialists were able to provide training, promote the use of RCA, help categorize risk factors associated with maternal mortality, and act as a resource. Most importantly, the low resource physician leaders with support from the MFM specialists were able to implement feasible and achievable improvement projects derived from the systematic maternal mortality review aimed at reducing maternal mortality in their local environment. For example, the site in Ghana worked on system to provide feedback to referring health centers, the care team and the administrative staff. The Uganda site developed an emergency cupboard for cesarean delivery supplies as a result of the mortality, the site in India reviewed the process of close monitoring of the 4th stage of labor and the Haiti site ensured that wall oxygen was available in the maternity unit. Our experience can be used to make some suggestions for international collaboration to reduce maternal mortality as follows:

1) The first step requires that the physicians in high resource communities interested in global health and maternal mortality expand their knowledge on all aspects of maternal mortality including global scope, systematic maternal mortality reviews, root cause analysis process including fish bone diagram analysis, standard protocols to obstetrical emergencies, quality improvement projects implementation and current initiatives. Maternal mortality is a complex problem involving complex system problems; the motivated physician may need to learn the LEAN or Kaizen principles. The term 'lean thinking or Kaizen principles' is based on the production philosophy, which evolved at Toyota to understand processes in order to identify and analyze problems. Lean thinking has been applied successfully in a wide variety of healthcare settings. The four general components of lean thinking in use are: 


\section{Global Journal of Reproductive Medicine}

a. methods to understand processes in order to identify and analyze problems

b. methods to organize more effective and/or efficient processes

c.methods to improve error detection, relay information to problem solvers, and prevent errors from causing harm

d. methods to manage change and solve problems with a scientific approach [13].

2) The trained physicians can then identify a physician leader in a low resource country to develop a professional and cooperative relationship. Identifying a compatible global physician partner can be challenging, and one may have to access experienced global health researchers, institutional and national programs.

3) Exchange visits are crucial to implementing a global health initiative. Ideally this should be bilateral. The high resource physician visits the low resource institution to get a scope of the local culture and challenges and thus be in a position to help train, guide and support maternal mortality reviews and initiatives. It is also important that the global health counterpart visit the high resource institution to learn, experience and be motivated.

4) Professional organizations in high resource countries should be encouraged to hold more global sessions to which emerging leaders are invited and sponsored. This will greatly increase motivation, mentoring and training of low resource physicians; increase collaborations and the sustainability of programs.

5) Constant and sustainable communication is the key to successful global collaboration especially between visits. In our programs we have used some form of telecommunication to have a regular conference at regular intervals. Ideally audiovisual telecommunications such as SKYPE, WEBEX are best, but because of limited Internet capacities in some countries we have had to depend on an audio only platform, which are more stable. The high-resource may need to be on a "on-call" schedule for access by phone or email in the advent of unusual or catastrophic obstetrical emergencies in the low resource country.

6) Finally, we believe that the collaboration should result in the identification and implementation of "specific, quantifiable and finite" quality improvement projects that can promote safe care and reduce maternal morbidity and mortality in the local region. Evidence shows the more specific the intervention as compared to broad interventions, the stronger the evidence that the intervention can help reduce maternal mortality [14].

In conclusion, we have shown that a group of MFM experts interested in global health, can develop mentoring and professional relationships with global counterparts that can facilitate systemic reviews of maternal mortality, lead to an awareness of risk factors and the implementation of preventive programs that can reduce maternal morbidity and mortality. We believe that it is possible to develop a group of specialists with a maternal health focus who can support local capacity building to facilitate local maternal mortality reviews and the implementation of appropriate prevention program

\section{References}

1. World Health Organization (1992) ICD 10: International statistical classification of diseases and related health problems. 10th revision. Geneva (Switzerland): World Health Organization.

2. Saucedo M, Deneux Tharaux C, Bouvier Colle MH (2013) For the French National Experts Committee on Maternal Mortality. Ten Years of Confidential Inquiries into Maternal Deaths in France. Obstet Gynecol 122(4): 752-760.

3. Rafael Lozano, Haidong Wang, Kyle J Foreman, Julie Knoll Rajaratnam, Mohsen Naghavi, et al. (2011) Progress towards Millennium Development Goals 4 and 5 on maternal and child mortality: an updated systematic analysis. Lancet 378: 1139-1165.

4. Elliot E, Menard MK (2013) Maternal Mortality, Time for National Action. Obstet Gynecol 122(4): 735-736.

5. Fassett WE (2011) Key performance outcomes of patient safety curricula: root cause analysis, failure mode and effects analysis, and structured communications skills. Am J Pharm Educ 75(8):164.

6. Hettinger AZ, Fairbanks RJ, Hegde S, Rackoff AS, Wreathall J, et al. (2013) An evidence-based toolkit for the development of effective and sustainable root cause analysis system safety solutions. J Healthc Risk Manag 33(2):11-20.

7. Thaddeus S, Maine D (1994) Too far to walk maternal mortality in context. Soc Sci Med 38(8): 1091-1110.

8. http://www.isixsigma.com/tools-templates/cause-effect/cause-andeffect-aka-fishbone-diagram/ accessed October 13th, 2013

9. Wong KC (2011) Using an Ishikawa diagram as a tool to assist memory and retrieval of relevant medical cases from the medical literature. J Med Case Rep 5:120.

10. Ghana Statistical Service (GSS), Ghana Health Service (GHS), and ICF Macro. 2009. Ghana Demographic and Health Survey 2008: Key Findings. Calverton, Maryland, USA: GSS, GHS, and ICF Macro.

11. Aboagye Patrick Kuma, Hailemichael Gebreselassie, Gloria Quansah Asare, Ellen MH Mitchell, Josephine Addy (2007) An assessment of the readiness to offer contraceptives and comprehensive abortion care in the Greater Accra, Eastern and Ashanti regions of Ghana. Chapel Hill, $\mathrm{NC}$, Ipas.

12. Kabakyenga J, O stergren P, Turyakira E, Pettersson K (2012) Influence of Birth Preparedness, Decision-Making on Location of Birth and Assistance by Skilled Birth Attendants among Women in SouthWestern Uganda. PLoS ONE 7(4): e35747.

13. Mazzocato P, Savage C, Brommels M, Aronsson H, Thor J (2010) Lean thinking in healthcare: a realist review of the literature. Qual Saf Health Care 19(5): 376-382.

14. Fortney JA, Leong M (2009) Saving mother's lives programs that work. Clin Obstet Gynecol 52(2): 224-236. 
This work is licensed under Creative Commons Attribution 4.0 License DOI: 10.19080/GJORM.2019.07.555701

\begin{tabular}{l} 
Your next submission with Juniper Publishers \\
will reach you the below assets \\
- Quality Editorial service \\
- Swift Peer Review \\
- Reprints availability \\
- E-prints Service \\
- Manuscript Podcast for convenient understanding \\
- Global attainment for your research \\
- Manuscript accessibility in different formats \\
( Pdf, E-pub, Full Text, Audio) \\
- Unceasing customer service \\
Track the below URL for one-step submission \\
https://juniperpublishers.com/online-submission.php \\
\hline
\end{tabular}

